\title{
A travelling wave approach to a multi-agent system with a path-graph topology
}

\author{
Dan Martinec*, Ivo Herman, and Michael Šebek ${ }^{1}$ \\ Department of Control Engineering, Czech Technical University in Prague, Prague, Czech Republic
}

\begin{abstract}
The paper presents a novel approach for the analysis and control of a multi-agent system with non-identical agents and a pathgraph topology. With the help of irrational wave transfer functions, the approach describes the interaction among the agents from the 'local' perspective and identify the travelling waves in the multi-agent system. The local treatment of the multi-agent system is complementary to the traditional 'overall' approach. It is shown that the different dynamics of the agents creates a virtual boundary that causes a partial reflection of the travelling waves. Undesired effects due to the reflection of the waves, such as amplification/attenuation, long transient or string instability, can be compensated by the feedback controllers introduced in this paper. A set of functions in MATLAB, which allows numerical simulation of the proposed approach, have been made available on MATLAB Central.
\end{abstract}

Keywords: multi-agent system, travelling waves, wave transfer function, path graph, irrational transfer function

\section{Introduction}

\subsection{Motivation}

A path graph is one of the simplest and most studied interaction topology of a multi-agent system. It represents interactions in a system where each agent, except of the first and last ones, interacts with two neighbouring agents. Although a path-graph topology is relatively simple, it serves, for instance, as a model of vehicular platoons [9], [33], discretized flexible structures [29] or [7], or a spatially-discretized models of long electrical transmission lines [6]. A path graph is also called a chain graph, see for instance [8] or [4].

The interaction between the agents causes that a change in the agent output, for instance, its position, of even a remote agent affects all the other agents. The propagation of the change in the multi-agent system can be described with the help of travelling waves. We will illustrate it on an example of a multiagent system with identical agents and a path-graph topology. If the first agent changes its output, then all following agents sequentially respond to this change. If we study their response from the local point of view, see [22], [17] or [30], we can notice that the change is propagated as a wave. The wave departs from the first agent and travels along the system to the last agent, where it reflects and travels back. When it reaches the first agent, it reflects back again. These two reflections on the system boundaries significantly prolong the settling time.

The same phenomenon is apparent if the agents are nonidentical, for instance, if the agents have different dynamics, or different controllers. In fact, the travelling wave is partially reflected on non-identical agents inside the multi-agent system,

\footnotetext{
${ }^{*}$ Corresponding author.

${ }^{1}$ E-mail addresses: martinec.dan@fel.cvut.cz (D. Martinec), ivo.herman@fel.cvut.cz (I. Herman), sebekm1@fel.cvut.cz (M. Šebek).
}

see [18]. We can imagine this behaviour as the reflection of the wave if it encounters a boundary between two media of different properties, see for instance [10]. However, a wave reflection is usually an undesired effect since it prolongs the settling time. One way to avoid the reflections inside the system is to force the agents to be identical, which is usually impractical, or even impossible. On the other hand, a wave description allows us to design a feedback controller that compensates the different dynamics of the agents, which shortens the settling time.

In this paper, we aim to provide the mathematical description of the travelling waves propagating along a multi-agent system with non-identical agents and path-graph topology. The underlying questions are: How do the different dynamics of the agents affect the travelling wave? How to mathematically describe this effect? How to compensate it?

\subsection{Laplacian and transfer function approaches to path graphs}

A popular tool for the convergence analysis of multi-agent systems comes from the algebraic graph theory, for instance, the spectral properties of the Laplacian matrix, see [20], [25] and [27] for a thorough overview.

The analysis of a multi-agent system with a path-graph topology focuses on the scaling of the magnitude of frequency response with the increasing size of the system, see e.g., [21], [11] and [12], as well as the locations of poles and zeros affecting the input-output behaviour of a path graph, see e.g., [2]. Another topic of interest is the scaling of the transient with the size of the system, see e.g., [34], [3] and [15].

The system analysis is related to vehicular-platoon control, where the string stability is widely used as an analytic measure of system performance. Although the string stability usually refers to vehicle-following applications, see e.g., [9] and [26], it is defined for an arbitrary interconnected system [32]. 
These approaches are well suited for the analysis of overall system behaviour such as the asymptotic stability. However, they do not treat a local behavior of the agents, for instance, how the disturbance is locally propagated from an agent to its immediate neighbours. This local-performance analysis can be carried out with the travelling-wave approach proposed in the paper.

\subsection{Transfer-function approach to travelling waves}

The transfer-function approach has recently been revisited in a series of papers for lumped models (see [22] and [35]) and for continuous flexible structures (see for the overview [30]). The travelling wave approach has also been applied to vibration control [19] and it seems to be related to the impedance matching in the power networks, see for instance [14].

The description of the travelling waves is based on the assumption of the spatial causality, that is, the boundary condition does not affect the wave travelling towards it. This assumption is applied to the description of infinite number of agents in the multi-agent system, which results in the description by irrational transfer function. The analyses of irrational and rational transfer functions differ in several aspects, see [5] for a thorough overview. For instance, despite many examples of the inverse Laplace transform of irrational transfer functions, see [1], it is difficult to find an exact impulse response for some of them. We note that the travelling wave approach can be used to describe even a system with finite number of agents by considering the boundary conditions.

This paper continues in the research started in [17], where the wave propagation in a platoon of identical vehicles is described. The platoon can be viewed as a chain of identical mechanicallyunconnected agents. The virtual interconnection is created by the controllers onboard each vehicle. A natural extension of this model is to consider a chain of non-identical agents. The first step in the treatment of such a model from the travelling-wave point of view is given in [23], where the description is limited to a mass-spring model. We generalize it by considering an arbitrary dynamics of the agents and their controllers. The preliminary results are presented in [18], where we introduce the soft boundary in a chain of vehicles. Here, we follow this concept of boundaries in a multi-agent system and introduce the second fundamental type of boundary, the hard boundary. Although the boundaries are virtual in nature, they principally affect the overall system behaviour. We present some fundamental properties of the boundaries and design wave-absorbing controllers for both types of boundaries.

The main contributions of the paper are: i) mathematical description of the travelling waves in a multi-agent system with non-identical agents and a path-graph topology given by Theorems 1 and 2 ii) a local performance analysis of the multi-agent system by the analysis of the wave amplification determined by the DC gains from Lemma 2, iii) a design of a controller that prevents a reflection of the travelling wave, which shortens the transient of the system, described in Theorems 3 and 4 , and iv) a set of functions in MATLAB, see WaveBox [16], which allows numerical simulations for irrational transfer functions.

\section{Mathematical preliminaries}

\subsection{Local control law}

We consider a multi-agent system with a path-graph interaction topology, for instance, a platoon of vehicles on a highway. The goal of the system is to drive along a line with equal distances between the agents.

The dynamics of agents is described by a linear single-inputsingle-output model, the transfer function $P_{n}(s)$, where $n$ is index of the agent. The output, $X_{n}(s)$, is described as

$$
X_{n}(s)=P_{n}(s) U_{n}(s),
$$

where $U_{n}(s)$ is the input to the agent generated by a local control law of the agent. The local control law of the agent aims to equalize its output with the outputs of the two neighbouring agents. It is modelled as

$$
\begin{aligned}
U_{n}(s) & =C_{\mathrm{f}, n}(s)\left(X_{n-1}(s)-X_{n}(s)\right)+C_{\mathrm{r}, n}(s)\left(X_{n+1}(s)-X_{n}(s)\right) \\
& +C_{\mathrm{f}, n}(s) W_{\mathrm{f}, n}(s)+C_{\mathrm{r}, n}(s) W_{\mathrm{r}, n}(s)
\end{aligned}
$$

where $C_{\mathrm{f}, n}(s)$ and $C_{\mathrm{r}, n}(s)$ are transfer functions of the controller of the front and rear agents, respectively, $W_{\mathrm{f}, n}(s)$ and $W_{\mathrm{r}, n}(s)$ are inputs to the agent. We assume that the inputs are equal to zero unless we specify them otherwise. We consider that each agent may have a different model as well as a different set of controllers and denote the front agent transfer function (ATF) and rear ATF by $M_{\mathrm{f}, n}(s)=P_{n}(s) C_{\mathrm{f}, n}(s)$ and $M_{\mathrm{r}, n}(s)=P_{n}(s) C_{\mathrm{r}, n}(s)$, respectively. The resulting model of the $n$th agent, assuming zero initial conditions, is shown in Fig. 1] and described as

$$
\begin{aligned}
X_{n}(s) & =M_{\mathrm{f}, n}(s)\left(X_{n-1}(s)-X_{n}(s)\right)+M_{\mathrm{r}, n}(s)\left(X_{n+1}(s)-X_{n}(s)\right) \\
& +M_{\mathrm{f}, n}(s) W_{\mathrm{f}, n}(s)+M_{\mathrm{r}, n}(s) W_{\mathrm{r}, n}(s)
\end{aligned}
$$

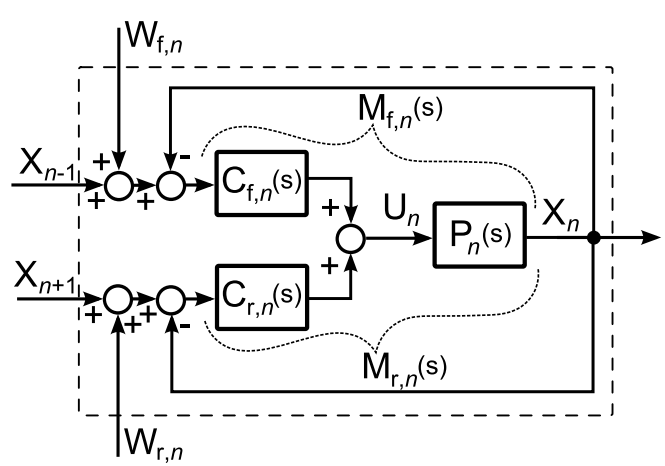

Figure 1: The model of $n$th agent.

The first agent is described as

$X_{1}(s)=M_{\mathrm{f}, 1}(s)\left(W_{\mathrm{f}, 1}(s)-X_{1}(s)\right)+M_{\mathrm{r}, 1}(s)\left(X_{2}(s)-X_{1}(s)\right)$,

where $W_{\mathrm{f}, 1}(s)=X_{\text {ref }}(s)$ is the external input to the multi-agent system, which represents the reference output of the multiagent system. The last agent, the rear-end agent, $(n=N)$ of the system is described as

$$
X_{N}(s)=M_{\mathrm{f}, N}(s)\left(X_{N-1}(s)-X_{N}(s)\right) .
$$




\subsection{Wave transfer function}

This section briefly summarize the main results of [17], where the wave propagation along a platoon of identical vehicles, that means $M_{\mathrm{f}, n}(s)=M_{\mathrm{r}, n}(s)=M(s)$, is studied. The results are also applicable for any multi-agent system with a path-graph topology.

The key idea of the Wave transfer function (WTF) approach is that the output of the $n$th agent in a multi-agent system with path-graph topology is described by two components, $A_{n}(s)$ and $B_{n}(s)$, that represent two waves propagating along the system in the forward and backward directions, respectively. The WTF describes how the wave propagates in the system. It is defined by the transfer function $G(s)=X_{n+1}(s) / X_{n}(s)$ for $N \rightarrow \infty$, see Section 3.1 in [17]. When $W_{\mathrm{f}, n}=W_{\mathrm{r}, n}=0$, the multi-agent system with identical agents is described as

$$
\begin{aligned}
X_{n}(s) & =A_{n}(s)+B_{n}(s), \\
A_{n+1}(s) & =G(s) A_{n}(s), \\
B_{n}(s) & =G(s) B_{n+1}(s),
\end{aligned}
$$

where $n \in\langle 1, N-1\rangle, G(s)$ is the WTF and $G^{-1}(s)=1 / G(s)$,

$$
\begin{aligned}
G(s) & =\frac{1}{2} \alpha(s)-\frac{1}{2} \sqrt{\alpha^{2}(s)-4}, \\
G^{-1}(s) & =\frac{1}{2} \alpha(s)+\frac{1}{2} \sqrt{\alpha^{2}(s)-4},
\end{aligned}
$$

with $\alpha(s)=2+1 / M(s)$, or, alternatively, $\alpha(s)=G(s)+G^{-1}(s)$.

The multi-agent system with identical agents and a pathgraph topology has only two boundaries (Fig. 2). The forcedend boundary, located at the first agent, is described by

$$
A_{1}(s)=G(s) W_{\mathrm{f}, 1}(s)-G^{2}(s) B_{1}(s) .
$$

and the free-end boundary, located at the rear-end agent, is described by

$$
B_{N}(s)=G(s) A_{N}(s)
$$

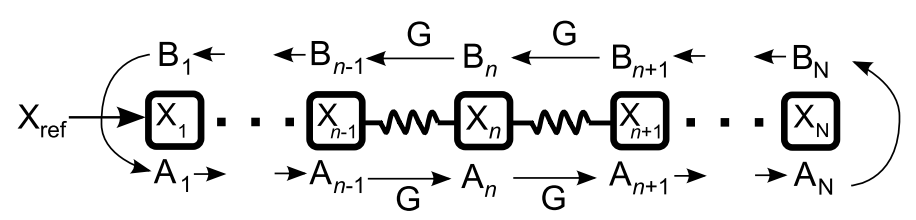

Figure 2: Scheme of a multi-agent system with a path-graph topology. The squares are agents with local dynamics described by (3) and (5). The virtual connections between the agents, which are created by the local control law, are illustrated by springs. All agents are identical.

For both types of path-graph boundaries, a wave-absorbing controller can be designed to prevent a reflection of the incident wave. The controller absorbs the travelling wave by calculating the incident part of the wave and adding this part to its output. Mathematically, the input to the first agent changes to

$$
\begin{aligned}
W_{\mathrm{f}, 1}(s) & =X_{\mathrm{ref}}(s)+G(s) B_{1}(s) \\
& =G(s) X_{1}(s)+\left(1-G(s)^{2}\right) X_{\mathrm{ref}}(s) .
\end{aligned}
$$

This concludes the brief summary of [17].

We denote the WTFs of two agents with different dynamics by $G(s)$ and $H(s)$ and symbolize them by blue and red colors in the following figures, respectively.

\subsection{Additional input to the agent}

Now, we generalize (6) for the case with a non-zero input. For now, we consider only the input $W_{\mathrm{r}, n}$ while $W_{\mathrm{f}, n}=0$. Hence, the model (3) of the $n$th agent is

$$
\begin{aligned}
X_{n}(s) & =M_{\mathrm{f}, n}(s)\left(X_{n-1}(s)-X_{n}(s)\right)+M_{\mathrm{r}, n}(s)\left(X_{n+1}(s)-X_{n}(s)\right) \\
& +M_{\mathrm{r}, n}(s) W_{\mathrm{r}, n}(s) .
\end{aligned}
$$

In this case, the input $W_{\mathrm{r}, n}(s)$ generates a wave that propagates in the multi-agent system in the same manner as describe (7) and (8). However, (6) is changed as follows

$$
X_{n}(s)=G(s) A_{n-1}(s)+G(s) B_{n+1}(s)+T_{\mathrm{r}, n}(s) W_{\mathrm{r}, n}(s),
$$

where $T_{\mathrm{r}, n}(s)=X_{n}(s) / W_{\mathrm{r}, n}(s)$ for $N \rightarrow \infty$. We are interested in finding $T_{\mathrm{r}, n}(s)$, hence, we substitute (7) and (8) into (14) and get

$$
\begin{aligned}
T_{\mathrm{r}, n} W_{\mathrm{r}, n} & =M_{\mathrm{f}, n}\left(G T_{\mathrm{r}, n} W_{\mathrm{r}, n}-T_{\mathrm{r}, n} W_{\mathrm{r}, n}\right) \\
& +M_{\mathrm{r}, n}\left(G T_{\mathrm{r}, n} W_{\mathrm{r}, n}-T_{\mathrm{r}, n} W_{\mathrm{r}, n}\right)+M_{\mathrm{r}, n} W_{\mathrm{r}, n} .
\end{aligned}
$$

Rearranging it gives

$$
T_{\mathrm{r}, n}(s)=\frac{M_{\mathrm{r}, n}(s)}{1+M_{\mathrm{f}, n}(s)+M_{\mathrm{r}, n}(s)-M_{\mathrm{f}, n}(s) G(s)-M_{\mathrm{r}, n}(s) G(s)} .
$$

Analogously, it can be shown that the transfer function $T_{\mathrm{f}, n}(s)=$ $X_{n}(s) / W_{\mathrm{f}, n}$ for a non-zero input $W_{\mathrm{f}, n}$.

\subsection{Analyzed properties}

The DC gain describing the steady-state amplification of a system, and $L_{2}$ string stability describing the amplification of disturbance in a system with the path-graph topology, are important analytical tools of system analysis. They are defined as follows.

Definition 1. The DC gain $\kappa_{\mathrm{G}}$ of the transfer function $G(s)$ is defined as $\kappa_{\mathrm{G}}=\lim _{s \rightarrow 0} G(s)$.

Definition 2. (From [9]) A system is called $L_{2}$ string stable if there is an upper bound on the $L_{2}$-induced system norm of $T_{0, n}$ that does not depend on the number of agents, where $T_{0, n}$ is the transfer function from $X_{\text {ref }}(s)$ to $X_{n}(s)$.

\section{Soft and hard boundaries}

\subsection{Mathematical definition of the boundaries}

We consider that the multi-agent system consists of nonidentical agents. In general, we can distinguish between three cases: i) $M_{\mathrm{r}, n} \neq M_{\mathrm{f}, n+1}$, ii) $M_{\mathrm{f}, n} \neq M_{\mathrm{r}, n}$, and iii) a combination of i) and ii). All three cases causes a partial reflection of the travelling wave. Therefore, we can consider them as boundaries for the wave. First, let us focus on the boundaries caused by i) and ii). 
Definition 3. The soft boundary is a virtual boundary between two agents, indexed $s$ and $s+1$, with the following property

$$
M_{\mathrm{r}, s}(s) \neq M_{\mathrm{f}, s+1}(s) .
$$

The soft boundary is, for instance, located in a platoon of non-identical vehicles governed by the same symmetric bidirectional control law, see [18], or in a mass-spring model with identical springs but non-identical masses.

The second type of boundary is defined as follows.

Definition 4. The hard boundary is a virtual boundary located at the $h$ th agent with the property

$$
M_{\mathrm{f}, h}(s) \neq M_{\mathrm{r}, h}(s) .
$$

The hard boundary is, for instance, located in a platoon of identical vehicles governed by the asymmetric bidirectional control, or in a mass-spring model with identical masses but different springs, see Fig. 3

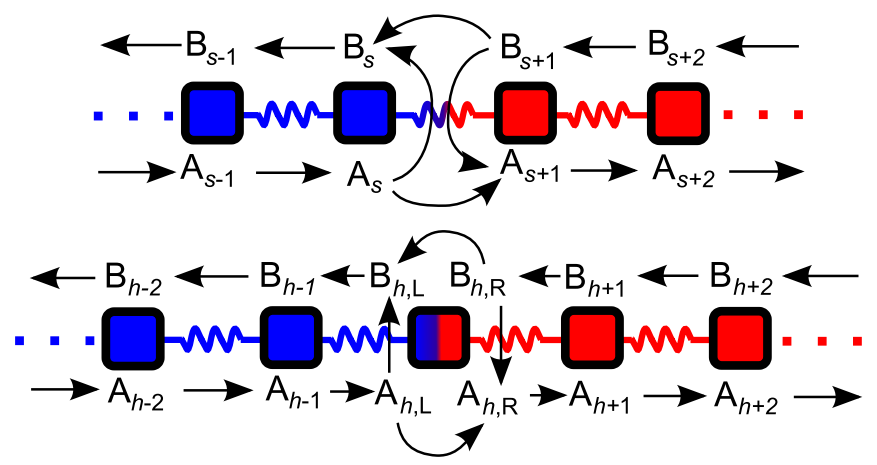

Figure 3: Scheme of a multi-agent system with the soft and hard boundaries in top and bottom, respectively. The blue and red squares are agents with the WTFs of $G(s)$ and $H(s)$, respectively, defined in (23) and (26). The virtual connections between the agents are illustrated by springs. The blue-red spring is the soft boundary and the blue-red square is the hard boundary.

The adjective 'hard' emphasizes the fact that the hard boundary is located at an agent, in contrast to the soft boundary, located between two agents. To distinguish between the incident, transmitted and reflected waves at the hard boundary, we decompose $X_{h}$ to the hard-boundary wave components as

$$
X_{h}(s)=A_{h, \mathrm{~L}}(s)+B_{h, \mathrm{~L}}(s)=A_{h, \mathrm{R}}(s)+B_{h, \mathrm{R}}(s),
$$

where the indexes $\mathrm{L}$ and $\mathrm{R}$ denote the wave components that are next to the left and right sides of the boundary, respectively. Changing the output of the agent with the hard boundary initiates waves propagating in both directions with different dynamics. This is treated in following technical Lemma.

Lemma 1. If there is no other boundary next to the hardboundary agent, then

$$
\begin{aligned}
& A_{h, \mathrm{~L}}(s)=G(s) A_{h-1}(s), \quad B_{h, \mathrm{~L}}(s)=G^{-1}(s) B_{h-1}(s), \\
& A_{h, \mathrm{R}}(s)=H^{-1}(s) A_{h+1}(s), \quad B_{h, \mathrm{R}}(s)=H(s) B_{h+1}(s),
\end{aligned}
$$

where

$$
G(s)=\frac{1}{2} \alpha_{1}-\frac{1}{2} \sqrt{\alpha_{1}^{2}-4}, \quad H(s)=\frac{1}{2} \alpha_{2}-\sqrt{\alpha_{2}^{2}-4},
$$

$\alpha_{1}=2+1 / M_{\mathrm{f}, h}(s)$ and $\alpha_{2}=2+1 / M_{\mathrm{r}, h}(s)$.

Proof. The proof is the same as that in Section 3.1 [17]. In this case, two different sets of continued fractions can be found, one converges to $G$ and $G^{-1}$, and the other to $H$ and $H^{-1}$.

The third case introduced at the beginning of this section, that is the combination of the soft and hard boundaries, can be treated by generalizing Lemma 1 , which is treated in Section 5.1

\subsection{Mathematical description of the boundaries}

Theorem 1. (From [18]) A soft boundary is in the Laplace domain described by the following four boundary-transfer functions (BTFs),

$$
\begin{gathered}
T_{\text {aa }}=\frac{A_{s+1}}{A_{s}}=\frac{H-H G^{2}}{1-H G}, \quad T_{\mathrm{ba}}=\frac{A_{s+1}}{B_{s+1}}=\frac{H G-H^{2}}{1-H G}, \\
T_{\mathrm{bb}}=\frac{B_{s}}{B_{s+1}}=\frac{G-H^{2} G}{1-H G}, \quad T_{\mathrm{ab}}=\frac{B_{s}}{A_{s}}=\frac{H G-G^{2}}{1-H G},
\end{gathered}
$$

where

$$
G(s)=\frac{1}{2} \alpha_{1}-\frac{1}{2} \sqrt{\alpha_{1}^{2}-4}, \quad H(s)=\frac{1}{2} \alpha_{2}-\sqrt{\alpha_{2}^{2}-4},
$$

$\alpha_{1}=2+1 / M_{\mathrm{r}, s}(s)$ and $\alpha_{2}=2+1 / M_{\mathrm{f}, s+1}(s)$.

Proof. The proof is given in [18].

The interpretation of the theorem is as follows. If there is a wave travelling to the soft boundary from the left-hand side, then it is partially reflected from the boundary (described by $T_{\mathrm{ab}}$ ) and partially transmitted through the boundary (described by $T_{\text {aa }}$ ). Likewise, if the wave travels from the opposite side, then the transfer functions $T_{\mathrm{ba}}$ and $T_{\mathrm{bb}}$ represent the respective waves. Mathematically,

$$
\begin{aligned}
X_{s}(s) & =G(s)\left(1+T_{\mathrm{ab}}(s)\right) A_{s-1}(s)+T_{\mathrm{bb}}(s) B_{s+1}(s), \\
X_{s+1}(s) & =H(s)\left(1+T_{\mathrm{ba}}(s)\right) B_{s+2}(s)+T_{\mathrm{aa}}(s) A_{s}(s) .
\end{aligned}
$$

The forced-end boundary is an example of the soft boundary. Substituting $G=0$ into 24 and 25 gives $T_{\text {aa }}=H, T_{\text {ba }}=-H^{2}$ and $T_{\mathrm{bb}}=T_{\mathrm{ab}}=0$.

Theorem 2. The BTFs describing the hard boundary in the Laplace domain are

$$
\begin{array}{ll}
T_{\mathrm{AA}}=\frac{A_{h, \mathrm{R}}}{A_{h, \mathrm{~L}}}=\frac{(1+G)(1-H)}{1-H G}, & T_{\mathrm{BA}}=\frac{A_{h, \mathrm{R}}}{B_{h, \mathrm{R}}}=\frac{H-G}{1-H G}, \\
T_{\mathrm{BB}}=\frac{B_{h, \mathrm{~L}}}{B_{h, \mathrm{R}}}=\frac{(1+H)(1-G)}{1-H G}, & T_{\mathrm{AB}}=\frac{B_{h, \mathrm{~L}}}{A_{h, \mathrm{~L}}}=\frac{G-H}{1-H G},
\end{array}
$$

where $G$ and $H$ are given by (23). 
Proof. From (3), the output of the agent can be rewritten as

$$
X_{h}(s)=T_{\mathrm{L}}(s) X_{h-1}(s)+T_{\mathrm{R}}(s) X_{h+1}(s),
$$

where $T_{\mathrm{L}}=M_{\mathrm{f}, h} /\left(1+M_{\mathrm{f}, h}+M_{\mathrm{r}, h}\right)$ and $T_{\mathrm{R}}=M_{\mathrm{r}, h} /\left(1+M_{\mathrm{f}, h}+M_{\mathrm{r}, h}\right)$. We combine (6), 21) and (22), and obtain

$$
\begin{aligned}
& X_{h-1}=A_{h-1}+B_{h-1}=G^{-1} A_{h, \mathrm{~L}}+G B_{h, \mathrm{~L}}, \\
& X_{h+1}=A_{h+1}+B_{h+1}=H A_{h, \mathrm{R}}+H^{-1} B_{h, \mathrm{R}} .
\end{aligned}
$$

Substituting (32) and (33) into (31) and using (20) for $X_{h}$, we have

$$
A_{h, \mathrm{~L}}+B_{h, \mathrm{~L}}=T_{\mathrm{L}}\left(G^{-1} A_{h, \mathrm{~L}}+G B_{h, \mathrm{~L}}\right)+T_{\mathrm{R}}\left(H A_{h, \mathrm{R}}+H^{-1} B_{h, \mathrm{R}}\right) .
$$

Rearranging (34) with respect to the hard-boundary wave components gives

$$
A_{h, \mathrm{~L}}\left(1-T_{\mathrm{L}} G^{-1}\right)+B_{h, \mathrm{~L}}\left(1-T_{\mathrm{L}} G\right)=B_{h, \mathrm{R}}\left(T_{\mathrm{R}} H^{-1}\right)+A_{h, \mathrm{R}} T_{\mathrm{R}} H .
$$

The four wave components are now reduced to three components by substituting $A_{h, \mathrm{R}}=A_{h, \mathrm{~L}}+B_{h, \mathrm{~L}}-B_{h, \mathrm{R}}$ into (35),

$$
B_{h, \mathrm{~L}}=B_{h, \mathrm{R}} \frac{T_{\mathrm{R}} H-T_{\mathrm{R}} H^{-1}}{T_{\mathrm{L}} G+T_{\mathrm{R}} H-1}+A_{h, \mathrm{~L}} \frac{1-T_{\mathrm{L}} G^{-1}-T_{\mathrm{R}} H}{T_{\mathrm{L}} G+T_{\mathrm{R}} H-1},
$$

or, alternatively, by substituting $B_{h, \mathrm{~L}}=A_{h, \mathrm{R}}+B_{h, \mathrm{R}}-A_{h, \mathrm{~L}}$,

$$
A_{h, \mathrm{R}}=A_{h, \mathrm{~L}} \frac{T_{\mathrm{L}} G-T_{\mathrm{L}} G^{-1}}{T_{\mathrm{L}} G+T_{\mathrm{R}} H-1}+B_{h, \mathrm{R}} \frac{1-T_{\mathrm{L}} G-T_{\mathrm{R}} H^{-1}}{T_{\mathrm{L}} G+T_{\mathrm{R}} H-1} .
$$

These formulas can be further simplified by expressing $T_{\mathrm{L}}$ and $T_{\mathrm{R}}$ in terms of $G$ and $H$. Specifically, $M_{\mathrm{f}, h}=\left(G+G^{-1}-2\right)^{-1}$ and $M_{\mathrm{r}, h}=\left(H+H^{-1}-2\right)^{-1}$. Substituting for them into 36 and 37) yields

$$
\begin{aligned}
& B_{h, \mathrm{~L}}=B_{h, \mathrm{R}} \frac{(1+H)(1-G)}{1-H G}+A_{h, \mathrm{~L}} \frac{G-H}{1-H G}, \\
& A_{h, \mathrm{R}}=A_{h, \mathrm{~L}} \frac{(1+G)(1-H)}{1-H G}+B_{h, \mathrm{R}} \frac{H-G}{1-H G},
\end{aligned}
$$

which proves the Theorem.

The interpretation of the theorem is as follows. A wave incident from the left side of the hard boundary (described by $A_{h, \mathrm{~L}}$ ) is partially reflected from the boundary (described by $T_{\mathrm{AB}}$ ) and partially transmitted through the boundary (described by $T_{\mathrm{AA}}$ ). For the wave incidenting from the opposite side (described by $B_{h, \mathrm{R}}$ ), the transfer functions are $T_{\mathrm{BA}}$ and $T_{\mathrm{BB}}$, respectively. The output of the hard-boundary agent can be expressed in two equivalent ways,

$$
\begin{aligned}
& X_{h}=G\left(1+T_{\mathrm{AB}}\right) A_{h-1}+H T_{\mathrm{BB}} B_{h+1}, \\
& X_{h}=G T_{\mathrm{AA}} A_{h-1}+H\left(1+T_{\mathrm{BA}}\right) B_{h+1} .
\end{aligned}
$$

The free-end boundary from (12) is an example of the soft boundary. In this case $H=0$, which gives $T_{\mathrm{AA}}=T_{\mathrm{BB}}=G$ and $T_{\mathrm{AB}}=T_{\mathrm{BA}}=0$.

For the case of identical agents, that means $G=H$, Theorems 1 and 2 yield $T_{\mathrm{aa}}=T_{\mathrm{bb}}=G, T_{\mathrm{ab}}=T_{\mathrm{ba}}=T_{\mathrm{AB}}=T_{\mathrm{BA}}=0$ and $T_{\mathrm{AA}}=T_{\mathrm{BB}}=1$, which is in agreement with $(6)-(8)$.

\subsection{Properties of the boundaries}

Although the above definitions and physical interpretations of the boundaries are different, they have some common features. For instance, they have bounded DC gains, or there is an inverse-reciprocity relation indicating that $T_{\mathrm{aa}}$ is closely related to $T_{\mathrm{BB}}$ rather than to $T_{\mathrm{AA}}$, while $T_{\mathrm{ab}}$ is related to $T_{\mathrm{BA}}$, and so on. More specifications are as follows.

Corollary 1. The soft and hard BTFs are related as follows,

$$
\begin{array}{ll}
T_{\mathrm{aa}}(s)=T_{\mathrm{BB}}(s)+G(s)-1, & T_{\mathrm{ba}}(s)=H(s) T_{\mathrm{AB}}(s), \\
T_{\mathrm{bb}}(s)=T_{\mathrm{AA}}(s)+H(s)-1, & T_{\mathrm{ab}}(s)=G(s) T_{\mathrm{BA}}(s), \\
T_{\mathrm{AA}}(s)=1+T_{\mathrm{AB}}(s), & T_{\mathrm{BB}}(s)=1+T_{\mathrm{BA}}(s), \\
T_{\mathrm{AA}}(s)+T_{\mathrm{BB}}(s)=2, & T_{\mathrm{AB}}(s)+T_{\mathrm{BA}}(s)=0 .
\end{array}
$$

Proof. By a straightforward application of Theorem 1 and Theorem 2

The amplitude of the wave that is reflected or transmitted through the boundary can be approximated by the DC gain of the BTFs. Usually, there is at least one integrator both in the front and rear ATFs allowing the agent to follow the ramp of $X_{\text {ref }}$, which can, for instance, represent that a vehicular platoon travelling with constant velocity. In this case, it holds.

Corollary 2. If there is at least one integrator in the front ATF and at least one integrator in the rear ATF, then the DC gains of the BTFs are related as follows

$$
\begin{gathered}
\kappa_{\mathrm{aa}}+\kappa_{\mathrm{bb}}=2, \quad \kappa_{\mathrm{ab}}+\kappa_{\mathrm{ba}}=0, \\
\kappa_{\mathrm{aa}}-\kappa_{\mathrm{ab}}=1, \quad \kappa_{\mathrm{bb}}-\kappa_{\mathrm{ba}}=1, \\
\kappa_{\mathrm{aa}}=\kappa_{\mathrm{BB}}, \quad \kappa_{\mathrm{ba}}=\kappa_{\mathrm{AB}}, \quad \kappa_{\mathrm{bb}}=\kappa_{\mathrm{AA}}, \quad \kappa_{\mathrm{ab}}=\kappa_{\mathrm{BA}},
\end{gathered}
$$

where $\kappa_{\mathrm{aa}}$ is the DC gain of $T_{\mathrm{aa}}, \kappa_{\mathrm{ab}}$ is the DC gain of $T_{\mathrm{ab}}$ etc.

Proof. Under the above assumptions, the DC gain of a WTF is equal to one, i.e. $\lim G(s)_{s \rightarrow 0}=1$ and $\lim H(s)_{s \rightarrow 0}=1$. Then, the proof is a straightforward application of Corollary 1 .

The particular values of $\kappa_{\mathrm{aa}}$ and $\kappa_{\mathrm{bb}}$ are given by the following lemma.

Lemma 2. Let both $M_{\mathrm{r}, n}$ and $M_{\mathrm{f}, n+1}$ have at least one integrator. If $M_{\mathrm{r}, n}$ and $M_{\mathrm{f}, n+1}$ have the same number of integrators, then the DC gains of the soft BTFs are

$$
\kappa_{\mathrm{aa}}=\frac{2}{\sqrt{\frac{n_{1,0} d_{2,0}}{n_{2,0} d_{1,0}}}+1}, \quad \kappa_{\mathrm{bb}}=\frac{2}{\sqrt{\frac{n_{2,0} d_{1,0}}{n_{1,0} d_{2,0}}}+1},
$$

where $n_{1,0} / d_{1,0}=\lim _{s \rightarrow 0} s^{k_{1}} M_{\mathrm{r}, n}, n_{2,0} / d_{2,0}=\lim _{s \rightarrow 0} s^{k_{2}} M_{\mathrm{f}, n+1}$, and $k_{1}$ and $k_{2}$ is the number of integrators in $M_{\mathrm{r}, n}$ and $M_{\mathrm{f}, n+1}$, respectively. If $M_{\mathrm{r}, n}$ has more integrators than $M_{\mathrm{f}, n+1}$, then

$$
\kappa_{\mathrm{aa}}=0, \quad \kappa_{\mathrm{bb}}=2 .
$$

If $M_{\mathrm{f}, n+1}$ has more integrators than $M_{\mathrm{r}, n}$, then

$$
\kappa_{\mathrm{aa}}=2, \quad \kappa_{\mathrm{bb}}=0 .
$$


Proof. Let us denote $\alpha_{1}=2+1 / M_{\mathrm{r}, n}=2+d_{1} / n_{1}$ and $\alpha_{2}=$ $2+1 / M_{\mathrm{f}, n+1}=2+d_{2} / n_{2}$. We will begin with deriving the DC gain $\kappa_{\mathrm{aa}}$ of the $T_{\text {aa }}$ transfer function.

$$
\kappa_{\mathrm{aa}}=\lim _{s \rightarrow 0} T_{\text {aa }}=\lim _{s \rightarrow 0} \frac{H-H G^{2}}{1-H G}=\lim _{s \rightarrow 0} \frac{1-G^{2}}{H^{-1}-G}=\frac{0}{0},
$$

since $\lim _{s \rightarrow 0} G=\lim _{s \rightarrow 0} H=1$ for at least one integrator in $M_{\mathrm{r}, n}$ and $M_{\mathrm{f}, n+1}$. Applying the L'Hopital's rule to (52) gives

$$
\kappa_{\mathrm{aa}}=\lim _{s \rightarrow 0} \frac{2 G}{H^{-2} H^{\prime}\left(G^{\prime}\right)^{-1}+1}=\lim _{s \rightarrow 0} \frac{2}{H^{\prime}\left(G^{\prime}\right)^{-1}+1},
$$

where the symbol' denotes the differentiation with respect to variable $s$. First, the differentiation of 26 yields

$$
G^{\prime}=\frac{1}{2} \alpha_{1}^{\prime}-\frac{1}{2} \frac{\alpha_{1} \alpha_{1}^{\prime}}{\sqrt{\alpha_{1}^{2}-4}} .
$$

The individual contributions to $\lim _{s \rightarrow 0} G^{\prime}$ are

$$
\lim _{s \rightarrow 0} \alpha_{1}^{\prime}=\lim _{s \rightarrow 0} \frac{d_{1}^{\prime} n_{1}-d_{1} n_{1}^{\prime}}{n_{1}^{2}}=\left\{\begin{array}{l}
\in \mathbb{R}, \text { if } k_{1}=1, \\
0, \text { if } k_{1}>1,
\end{array}\right.
$$

and

$$
\begin{aligned}
& \lim _{s \rightarrow 0} \frac{\alpha_{1}^{\prime}}{\sqrt{\alpha_{1}^{2}-4}}=\lim _{s \rightarrow 0} \frac{d_{1}^{\prime} n_{1}-d_{1} n_{1}^{\prime}}{n_{1} \sqrt{d_{1}^{2}+4 d_{1} n_{1}}} \\
& =\lim _{s \rightarrow 0} \frac{s^{\left(k_{1} / 2\right)}\left(s^{\left(k_{1} / 2\right)-1} k_{1} d_{1,0} n_{1}-s^{\left(k_{1} / 2\right)} d_{1,0} n_{1}^{\prime}\right)}{s^{\left(k_{1} / 2\right)} n_{1} \sqrt{s^{k_{1}} d_{1,0}^{2}+4 d_{1,0} n_{1}}} \\
& =\lim _{s \rightarrow 0} \frac{\left(s^{\left(k_{1} / 2\right)-1} k_{1} d_{1,0} n_{1}\right)}{n_{1} \sqrt{4 d_{1,0} n_{1}}}=\lim _{s \rightarrow 0} k_{1} s^{\left(k_{1} / 2\right)-1} \frac{1}{2} \sqrt{\frac{d_{1,0}}{n_{1,0}}}
\end{aligned}
$$

where $d_{1,0}=\lim _{s \rightarrow 0} s^{-k_{1}} d_{1}$ and $n_{1,0}=\lim _{s \rightarrow 0} n_{1}$. Moreover $\lim _{s \rightarrow 0} \alpha_{1}=2$. Similarly, $\lim _{s \rightarrow 0} H^{\prime}$ can be evaluated. Then

$$
\lim _{s \rightarrow 0} \frac{H^{\prime}}{G^{\prime}}=\lim _{s \rightarrow 0} s^{\left(k_{2}-k_{1}\right) / 2} \frac{k_{2}}{k_{1}} \sqrt{\frac{n_{1,0} d_{2,0}}{n_{2,0} d_{1,0}}},
$$

where $d_{2,0}=\lim _{s \rightarrow 0} s^{-k_{2}} d_{2}$ and $n_{2,0}=\lim _{s \rightarrow 0} n_{2}$. Finally, substituting (57) into 53 yields

$$
\kappa_{\mathrm{aa}}=\lim _{s \rightarrow 0} \frac{2}{s^{\left(k_{2}-k_{1}\right) / 2} \frac{k_{2}}{k_{1}} \sqrt{\frac{n_{1,0} d_{2,0}}{n_{2,0} d_{1,0}}}+1} .
$$

Therefore, if $M_{\mathrm{r}, n}$ and $M_{\mathrm{f}, n+1}$ have the same number of integrators $\left(k_{1}=k_{2}\right)$, then (58) simplifies to (49). If $k_{2}>k_{1}$, i.e., $M_{\mathrm{f}, n+1}$ have more integrators than $M_{\mathrm{r}, n}$, then (57) converges to zero and $\kappa_{\mathrm{aa}}=2$. In the opposite case, (57) diverges and $\kappa_{\mathrm{aa}}=0$. The DC gain $\kappa_{\mathrm{bb}}=2-\kappa_{\mathrm{aa}}$ is from (46).

Corollary 3. If there is at least one integrator in the front ATF and at least one integrator in the rear ATF, then the DC gains of the BTFs are bounded as

$$
\begin{aligned}
-1 & \leq \kappa_{\mathrm{ab}}, \kappa_{\mathrm{ba}}, \kappa_{\mathrm{AB}}, \kappa_{\mathrm{BA}} \leq 1, \\
0 & \leq \kappa_{\mathrm{aa}}, \kappa_{\mathrm{bb}}, \kappa_{\mathrm{AA}}, \kappa_{\mathrm{BB}} \leq 2 .
\end{aligned}
$$

Proof. The soft-boundary DC gains are treated by Lemma 2 and Corollary 2. The hard-boundary DC gains can be calculated using Corollary 2

\section{Controllers for the boundaries}

We now design a feedback controller compensating the fact that the agents are not identical. The motivation is to prevent the undesired reflection of the wave to shorten the settling time.

\subsection{The soft boundary controller}

A soft-boundary controller can be designed for various purposes, for instance, to prevent or modify a wave's transmission through the boundary. We now design an absorbing controller that prevents the reflection of a wave from the soft boundary. The derivation will be shown only for the left side of the boundary, since the derivation for its right side is analogous.

First, we add input $W_{\mathrm{r}, s}$ to the $s$ th agent. By the combination of 27) and (17), we get

$$
X_{s}=\left(1+T_{\mathrm{ab}}\right) A_{s}+T_{\mathrm{bb}} B_{s+1}+T_{\mathrm{r}, n}\left(1+T_{\mathrm{ab}}\right) W_{\mathrm{r}, s} .
$$

We want to design a controller that prevents the reflection of the wave travelling from the left, which is described by term $T_{\mathrm{ab}} A_{s}$. Therefore, we set $W_{\mathrm{r}, s}(s)=F_{\mathrm{f}, \mathrm{S}}(s) A_{s}(s)$, where $F_{\mathrm{f}, \mathrm{S}}(s)$ is a transfer function of a controller that prevents the reflection of the wave. To prevent the reflection, we eliminate term $T_{\mathrm{ab}} A_{s}$ by requiring

$$
T_{\mathrm{ab}}(s) A_{s}(s)+T_{\mathrm{r}, n}(s)\left(1+T_{\mathrm{ab}}(s)\right) F_{\mathrm{f}, \mathrm{S}}(s) A_{s}(s)=0,
$$

which is the constraint for the controller,

$$
F_{\mathrm{f}, \mathrm{S}}(s)=\frac{-T_{\mathrm{ab}}(s)}{T_{\mathrm{r}, n}(s)\left(1+T_{\mathrm{ab}}(s)\right)} .
$$

Substituting for $T_{\mathrm{ab}}(s)$ from (25) and for $T_{\mathrm{r}, n}(s)$ from (17), the 'absorbing' transfer function has a simple form

$$
F_{\mathrm{f}, \mathrm{S}}(s)=G(s)-H(s)
$$

It remains to specify $A_{s}$, which represents a wave incident on the soft boundary from the left. By $(6),(7)$ and $(8)$, we have

$$
A_{s}(s)=G\left(X_{s-1}-B_{s-1}\right)=G X_{s-1}-G^{2}\left(X_{s}-A_{s}\right),
$$

which leads to

$$
A_{s}(s)=\frac{G(s)}{1-G^{2}(s)} X_{s-1}(s)-\frac{G^{2}(s)}{1-G^{2}(s)} X_{s}(s) .
$$

Therefore, the left-side-absorbing control law $C_{\mathrm{L}, \mathrm{S}}$ is described by the consequential application of the above equations as

$$
\begin{aligned}
C_{\mathrm{L}, \mathrm{S}} & =M_{\mathrm{r}, s} W_{\mathrm{r}, s}=M_{\mathrm{r}, s} F_{\mathrm{f}, \mathrm{S}} A_{s} \\
& =M_{\mathrm{r}, s}(G-H) \frac{G}{1-G^{2}}\left(X_{s-1}-G X_{s}\right) .
\end{aligned}
$$




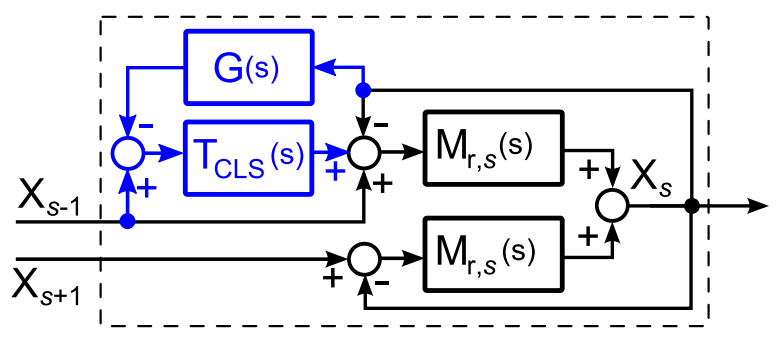

Figure 4: The model of sth agent with the left-side absorbing controller (highlighted in blue), where $T_{\mathrm{CLS}}=G(s)(G(s)-$ $H(s)) /\left(1-G^{2}(s)\right)$ from 67 . The front and rear ATFs are identical since, for now we assume only one soft boundary in the system as at the top of Fig. 3

The sth agent with implemented left-side absorbing controller is shown in Fig. 4

By modifying (61) the output of the sth agent with the leftand right-side absorbing controllers is

$$
\begin{aligned}
X_{s} & =\left(1+T_{\mathrm{ab}}\right) A_{s}+T_{\mathrm{bb}} B_{s+1}+F_{\mathrm{f}, \mathrm{S}} T_{\mathrm{r}, n}\left(1+T_{\mathrm{ab}}\right) A_{s} \\
& +T_{\mathrm{bb}} F_{\mathrm{r}, \mathrm{S}} T_{\mathrm{f}, n} B_{s+1}=G A_{s-1}+G B_{s+1},
\end{aligned}
$$

where $F_{\mathrm{r}, \mathrm{S}}(s)$ is equivalent of $F_{\mathrm{f}, \mathrm{S}}(s)$ for the right side of the soft boundary. Likewise, the output of the $(s+1)$ th agent with the absorbing controllers is

$$
X_{s+1}(s)=H(s) A_{s}(s)+H(s) B_{s+2}(s) .
$$

The results can be summarized in the following theorem.

Theorem 3. The control law preventing any wave to be reflected from the soft boundary is in the Laplace domain described as

$$
\begin{aligned}
X_{s} & =M_{\mathrm{r}, s}\left(X_{s-1}-2 X_{s}+X_{s+1}\right)+C_{\mathrm{L}, \mathrm{S}}, \\
X_{s+1} & =M_{\mathrm{f}, s+1}\left(X_{s}-2 X_{s+1}+X_{s+2}\right)+C_{\mathrm{R}, \mathrm{S}},
\end{aligned}
$$

where

$$
\begin{aligned}
C_{\mathrm{L}, \mathrm{S}} & =M_{\mathrm{r}, s} \frac{G(G-H)}{1-G^{2}}\left(X_{s-1}-G X_{s}\right), \\
C_{\mathrm{R}, \mathrm{S}} & =M_{\mathrm{f}, s+1} \frac{H(H-G)}{1-H^{2}}\left(X_{s+1}-H X_{s}\right)
\end{aligned}
$$

\subsection{The hard boundary controller}

Controlling the hard boundary is similar to that of the soft boundary in Section 4.1 Here, we only provide a brief description of the absorbing-controller design.

The output of the $h$ th agent from (40) controlled with additional input $W_{\mathrm{f}, h}(s)$ is

$$
X_{h}=\left(1+T_{\mathrm{AB}}\right) A_{h, \mathrm{~L}}+T_{\mathrm{BB}} B_{h, \mathrm{R}}+T_{\mathrm{f}, n} W_{\mathrm{f}, h},
$$

where $W_{\mathrm{f}, h}(s)=F_{\mathrm{f}, \mathrm{H}}(s) A_{h, \mathrm{~L}}(s)$ and $F_{\mathrm{f}, \mathrm{H}}(s)$ is a transfer function of a controller that prevents the reflection of a wave. To prevent the reflection of the wave travelling towards the hard boundary from the left, we set $T_{\mathrm{AB}} A_{h, \mathrm{~L}}=-F_{\mathrm{f}, \mathrm{H}} T_{\mathrm{f}, n} W_{\mathrm{f}, h}$. Hence,

$$
F_{\mathrm{f}, \mathrm{H}}(s)=-\frac{T_{\mathrm{AB}}(s)}{T_{\mathrm{f}, n}(s)}=\frac{(H(s)-G(s))(1-G(s))}{G(s)(1-H(s))} .
$$

The $A_{h, \mathrm{~L}}$ term represents the wave travelling towards the hard boundary from left, which is again computed by [66,

$$
A_{h, \mathrm{~L}}(s)=\frac{G(s)}{1-G^{2}(s)} X_{h-1}(s)-\frac{G^{2}(s)}{1-G^{2}(s)} X_{h}(s) .
$$

The left-side-absorbing control law is then

$$
C_{\mathrm{L}, \mathrm{H}}=M_{\mathrm{f}, h} F_{\mathrm{f}, \mathrm{H}} A_{h, \mathrm{~L}}=M_{\mathrm{f}, h} \frac{H-G}{(1+G)(1-H)}\left(X_{h-1}-G X_{h}\right)
$$

The design of the right-side absorbing controller is similar. When both controllers are implemented on the $h$ th agent, we get

$$
X_{h}(s)=A_{h, \mathrm{~L}}(s)+B_{h, \mathrm{R}}(s)=G(s) A_{h-1}(s)+H(s) B_{h+1}(s),
$$

where (44) have been considered.

Combining (78) and (20) gives $A_{h, \mathrm{~L}}=A_{h, \mathrm{R}}$ and $B_{h, \mathrm{~L}}=B_{h, \mathrm{R}}$. In words, the hard boundary between the wave components indexed by $\mathrm{L}$ and $\mathrm{R}$ is removed at the $h$ th agent and the wave transmits through the agent without being reflected.

The results can be summarized in the following theorem.

Theorem 4. The control law that prevents any wave to be reflected from the hard boundary is in the Laplace domain described as

$$
X_{h}=M_{\mathrm{f}, h}\left(X_{h-1}+X_{h}\right)+M_{\mathrm{r}, h}\left(X_{h+1}-X_{h}\right)+C_{\mathrm{L}, \mathrm{H}}+C_{\mathrm{R}, \mathrm{H}},
$$

where

$$
\begin{aligned}
& C_{\mathrm{L}, \mathrm{H}}=M_{\mathrm{f}, h} \frac{H-G}{(1+G)(1-H)}\left(X_{h-1}-G X_{h}\right), \\
& C_{\mathrm{R}, \mathrm{H}}=M_{\mathrm{r}, h} \frac{G-H}{(1+H)(1-G)}\left(X_{h+1}-H X_{h}\right) .
\end{aligned}
$$

By comparison of Theorems 3 and 4 we can see that we need to implement the wave absorber on two agents for the soft boundary but only on one agent for the hard boundary.

\subsection{Stability of the controllers}

To prove the stability of the control law, we first need to prove the stability of the WTF.

Lemma 3. If $M(s)$ is proper and has no CRHP (closed-right half plane) zeros and no CRHP poles, except for poles at the origin, and if

$$
1+4 M(j \omega)
$$

does not intersect the non-positive real axis for $\omega \in(0, \infty)$, then the wave transfer function $G(s)$ is asymptotically stable.

Proof. The proof is given in Appendix A

The stability of the proposed controllers is treated by the following Theorem.

Theorem 5. If the WTFs are asymptotically stable, then the multi-agent system with the path-graph topology and the control law from Theorem 3 or Theorem 4 is asymptotically stable. Furthermore, the control laws and the wave absorber, located on the first or rear-end agent make the multi-agent system $L_{2}$ string stable.

Proof. The proof is given in Appendix B 


\section{Numerical simulations of the soft boundary}

The numerical simulations are carried out with WaveBox, which is a set of functions and examples in MATLAB that numerically approximates WTFs and BTFs. The WaveBox also contains a set of examples that show the effect of boundaries and absorbers. Some of the examples are presented in this section. The WaveBox was written by the authors and is available at [16.

\subsection{Soft boundary performance}

The numerical simulations are carried out for a system with 8 agents described as

$$
\begin{gathered}
M_{\mathrm{f}, i}(s)=M_{\mathrm{r}, i}(s)=\frac{4 s+4}{s^{2}(s+4)}, \quad i=1,2,3,4, \\
M_{\mathrm{f}, j}(s)=M_{\mathrm{r}, j}(s)=\frac{s+1}{s^{2}(s+3)}, \quad j=5,6,7,8,
\end{gathered}
$$

which represents a double integrator agent with linear model of friction controlled by a PI controller. Therefore, we consider a multi-agent system with 8 agents and a soft boundary located between the 4th and 5 th agent.

The effect of the soft boundary is demonstrated in Fig. 5 where the wave-absorbing controllers on the first and rear-end agents are additionally implemented.

The performance of individual control strategies are shown in Fig. 6. Comparing the bottom-left and top-left panels, we can see that the soft-boundary absorber does not shorten the settling time if it is not combined with other absorbers on the first or rear-end agents. In the case of the absorber on the first agent (top-middle panel), the wave keeps reflecting between the soft boundary and the non-absorbing rear-end agent which prolongs the transient. The implementation of the soft-boundary absorber (bottom-middle panel) shortens the transient since it prevents the wave from being reflected back and forth. The absorbers implemented on both the first and rear-end agents (top-right panel) cause a change of the steady-state value, as predicted by Lemma 2. There are two possible ways to obtain a desired steady-state: a) overcompensate the input signal (see [18]), or b) implement the soft-boundary absorber (bottom-right panel).

Fig. 7 shows the comparison of the inputs to the fourth agent for (i) the multi-agent system with 8 identical agents (blue solid line), where each agent is described by (83), (ii) the multi-agent system with non-identical agents described by (83)-(84) without the soft-boundary absorber (green dashed line), and (iii) the multi-agent system as in (ii) but with the soft-boundary absorber (red pluses). The input for (i) and (ii) is described as $V_{\mathrm{I}}(s)=\left(X_{s-1}(s)+X_{s+1}(s)-2 X_{s}(s)\right)$, that means the output error. The input for (iii) is $V_{\mathrm{III}}(s)=V_{\mathrm{I}}(s)+C_{\mathrm{L}, \mathrm{S}}(s) / M_{\mathrm{r}, s}(s)$, that means the same as for (i) and (ii) plus the control law from (72). The wave absorbers on both the first and rear-end agents are implemented in all cases. We can see that the inputs to the fourth agent are the same for cases (i) and (iii). Therefore, the output of the wave-absorber is the difference between (ii) and (iii), which shows that the output of the controller is feasible.

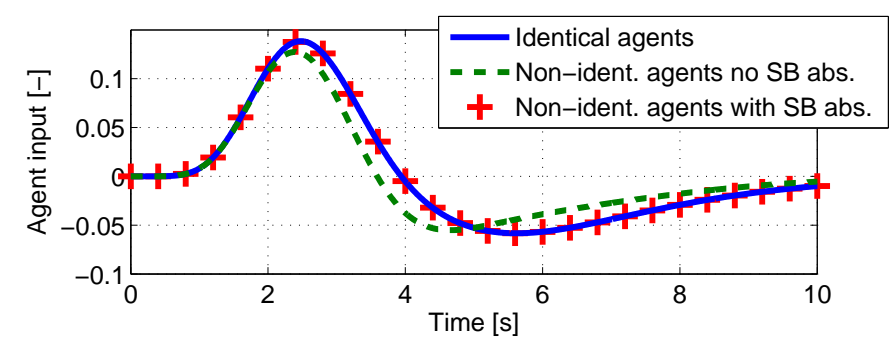

Figure 7: The comparison of the inputs to the fourth agent for three different multi-agent systems. The label 'SB abs.' stands for the soft-boundary absorber described by (67).

\subsection{Local effect of the DC gains}

A local effect of the BTF DC gains is demonstrated in Fig. 8 for first 120 seconds of the step response of a multi-agent system with a path-graph topology described by

$$
\begin{array}{r}
M_{\mathrm{f}, i}(s)=M_{\mathrm{r}, i}(s)=\frac{4 s+4}{s^{2}(s+4)}, \quad i=1,2, \ldots, 40, \\
M_{\mathrm{f}, j}(s)=M_{\mathrm{r}, j}(s)=\frac{s+k_{\mathrm{p}}}{s^{2}(s+3)}, \quad j=41,42, \ldots, 80 .
\end{array}
$$

Although the DC gain determines the steady state of the system, we can use the DC gain of $T_{\text {aa }}$ to approximate the output even before the whole multi-agent system reaches its steady state. We can also see that the transmitted wave is almost settled after approximately 50 seconds and the reflected wave returns back to the $41^{\text {st }}$ agent after about 100 seconds. Therefore the output of the $41^{\text {st }}$ agent between 50 and 100 seconds is approximated by $\kappa_{\mathrm{aa}}$ from 49 as follows

$$
\kappa_{\mathrm{aa}}=\frac{2}{\sqrt{\frac{4 \cdot 3}{k_{\mathrm{p}} \cdot 4}}+1},
$$

where we have substituted $n_{1,0}=4, d_{1,0}=4, n_{2,0}=k_{\mathrm{p}}$ and $d_{2,0}=3$. Therefore, the figure also numerically verifies Lemma 2 .

\section{A combination of soft and hard boundaries}

The soft and hard boundaries are two special cases of system boundaries. The two boundaries can be combined to form any type of complex boundary. The idea is to represent the transfer function of a complex boundary in terms of the soft- and hard-boundary BTFs. The combination of the two boundaries requires to relax the assumption for 21 and $(22)$, that is, there is no other boundary next to the hard or soft boundary.

The approach is demonstrated for the complex boundary in the multi-agent system, shown in Fig. 9 and described by

$$
\begin{aligned}
& M_{\mathrm{f}, i}(s)=M_{\mathrm{r}, i}(s)=\frac{4 s+4}{s^{2}(s+4)}, \quad i=1,2,4,5, \\
& M_{\mathrm{f}, 3}(s)=\frac{s+1}{s^{2}(s+3)}, \quad M_{\mathrm{r}, 3}(s)=\frac{4 s+4}{s^{2}(s+4)} .
\end{aligned}
$$


Time:0s
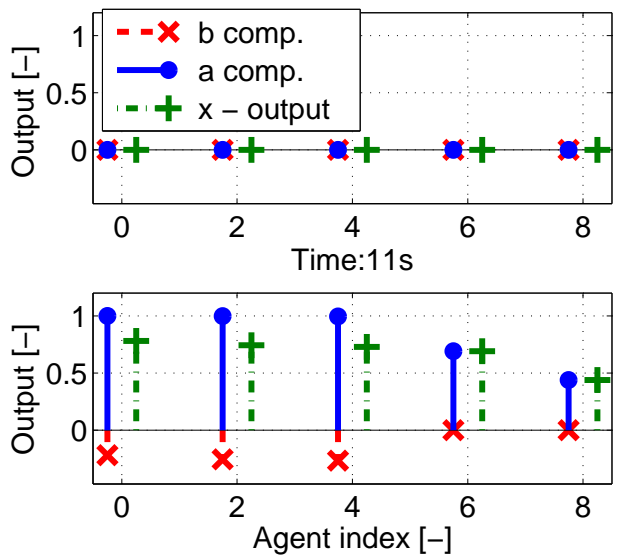

Time:2s
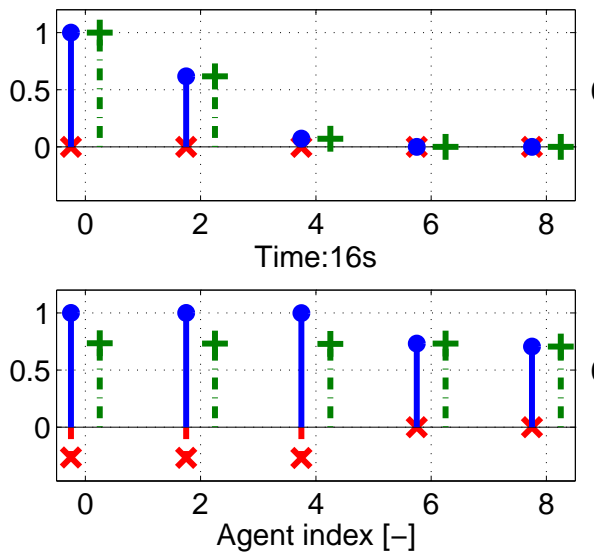

Time:6s

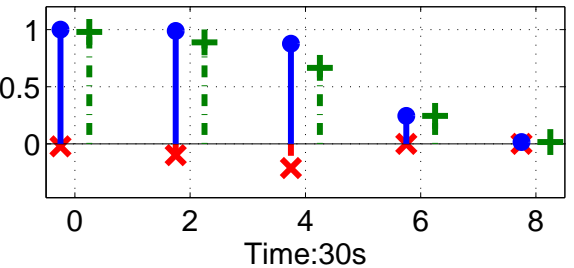

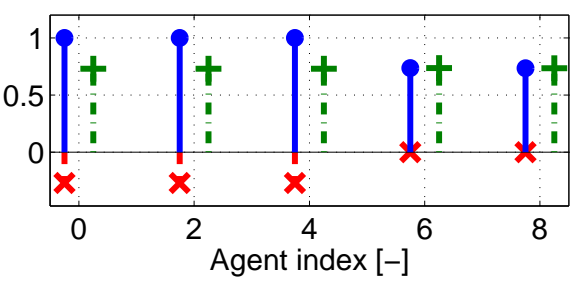

Figure 5: Simulation of the wave propagating in a multi-agent system with the path-graph topology defined by 83 - 84 . At the beginning, $t=0^{+} \mathrm{s}$, outputs of all agents are 0 except $x_{\text {ref }}(t)$, which is changed from 0 to 1 . At intermediate times, the wave travels to the soft boundary, where it is transmitted and attenuated by a factor $\kappa_{\mathrm{aa}}$ and reflected by a factor $\kappa_{\mathrm{ab}}$. As the wave propagates back to the fist agent, it forces the first four agents to change their output by $\kappa_{\mathrm{ab}}$ (negative value in this case). The waves are absorbed on 1 st and 8th agents by the wave-absorbing controllers. The blue circles and red crosses represent $a(t)$ and $b(t)$ components of the wave, respectively. The green plus signs stand for the outputs of the agents. The 0th agent is the input to the system from (13), i.e. $X_{0}(s)=W_{\mathrm{f}, 1}(s), A_{0}(s)=X_{\mathrm{ref}}(s)$ and $B_{0}(s)=G B_{1}(s)$.

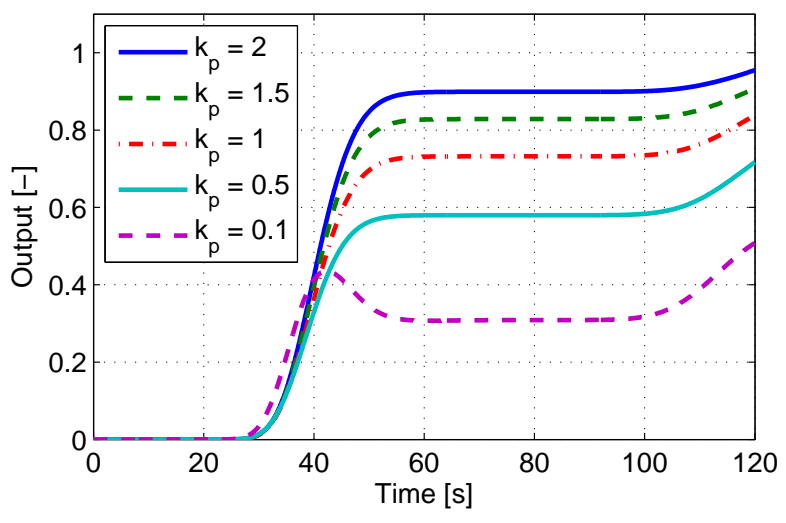

Figure 8: The numerical simulations of a multi-agent system described by $85-86$ for different values of $k_{\mathrm{p}}$. The output of the $41^{\text {st }}$ agent is shown.

The boundary is formed by the combination of the soft boundary located between the agents indexed 2 and 3 and the hard boundary located at the $3 \mathrm{rd}$ agent. This configuration violates the boundary conditions in (21) since now the wave travelling to $A_{3, \mathrm{~L}}$ and the wave travelling from $B_{3, \mathrm{~L}}$ transmit through the soft boundary. In view of Theorem 1 , we have

$$
\begin{aligned}
A_{3, \mathrm{~L}}(s) & =T_{\mathrm{aa}}(s) A_{2}(s)+T_{\mathrm{ba}}(s) B_{3, \mathrm{~L}}(s), \\
B_{2}(s) & =T_{\mathrm{ab}}(s) A_{2}(s)+T_{\mathrm{bb}}(s) B_{3, \mathrm{~L}}(s) .
\end{aligned}
$$

The second part of the complex boundary is composed of the hard boundary. Hence, by Theorem 2, we have

$$
\begin{aligned}
& A_{3, \mathrm{R}}(s)=\overline{T_{\mathrm{AA}}}(s) A_{3, \mathrm{~L}}(s)+\overline{T_{\mathrm{BA}}}(s) B_{3, \mathrm{R}}(s), \\
& B_{3, \mathrm{~L}}(s)=\overline{T_{\mathrm{AB}}}(s) A_{3, \mathrm{~L}}(s)+\overline{T_{\mathrm{BB}}}(s) B_{3, \mathrm{R}}(s),
\end{aligned}
$$

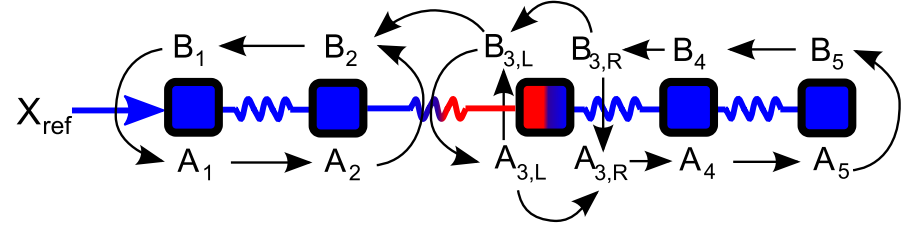

Figure 9: Scheme of the complex boundary between 2 nd and 3rd agents composed of the soft and hard boundaries next to each other in a multi-agent system described by $88-(89)$. The blue arrow in front of the first agent represents the input to the system.

where the overlined transfer functions are different from those in 29) and (30). The original transfer function $T_{\mathrm{AA}}$ describes the wave propagating from blue to red agent, while $\overline{T_{\mathrm{AA}}}$ describes the wave propagating in the opposite direction. Due to the same reasoning, $\overline{T_{\mathrm{AA}}}=T_{\mathrm{BB}}, \overline{T_{\mathrm{BB}}}=T_{\mathrm{AA}}, \overline{T_{\mathrm{AB}}}=T_{\mathrm{BA}}$ and $\overline{T_{\mathrm{BA}}}=T_{\mathrm{AB}}$ for this complex boundary.

The same procedure can be applied to the wave-absorbing controller. They can be combined to absorb the wave reflecting from boundaries of various complexity.

The independent validation of the WTF approach is shown in Fig. 10, where the results of the WTF approach is compared with the simulation by the state-space approach. We can see that the two results are identical. Although the validation is based on numerical simulation, we can validate it also analytically by finding the transfer function from $X_{\text {ref }}(s)$ to $\left(A_{2}(s)+B_{2}(s)\right)$. This transfer function is identical to the rational transfer function obtained by the state-space approach using 

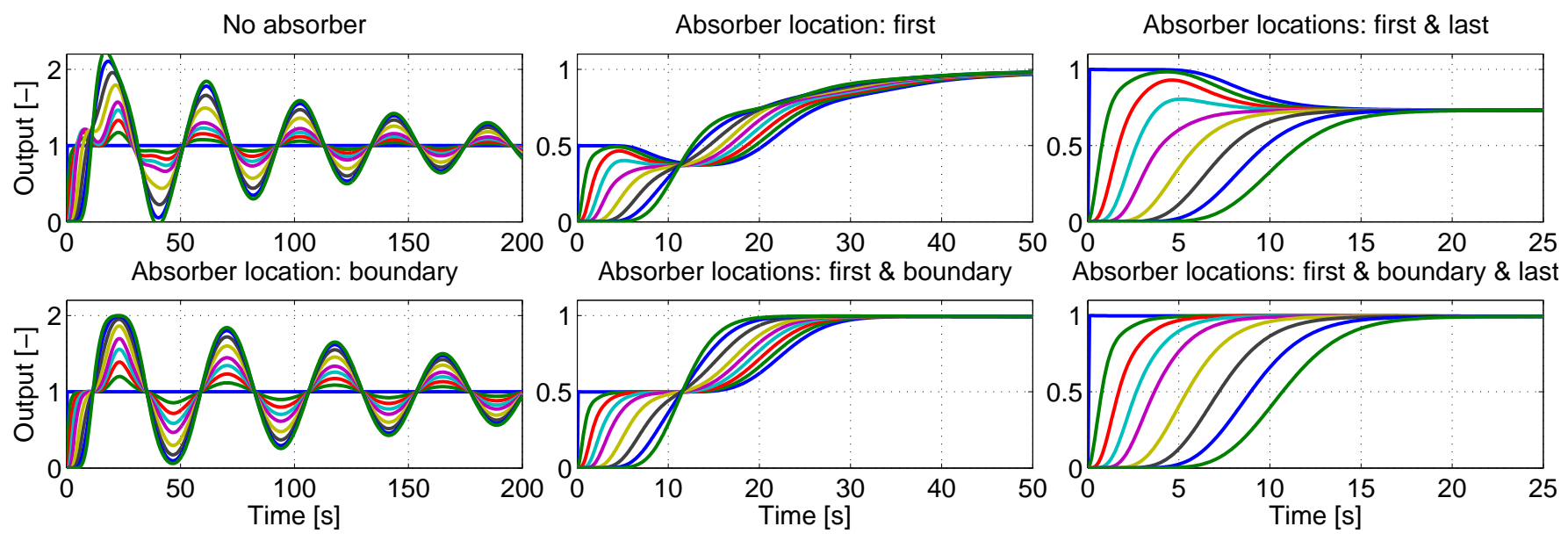

Figure 6: The performance comparison of individual control strategies for a multi-agent system with the soft boundary. The multiagent system is defined by 83 - 84 with $x_{\text {ref }}(0)=1$ and $x_{i}(0)=0$ for $i=1,2, \ldots, N$. The step responses of six individual control strategies are compared. The top-left: the system with no absorber; top-middle: the system with the absorber implemented on the first agent; top-right: the system with the absorbers implemented on the first and rear-end agents. In the bottom panels, the soft-boundary absorber between the agents 4 and 5 is additionally implemented.

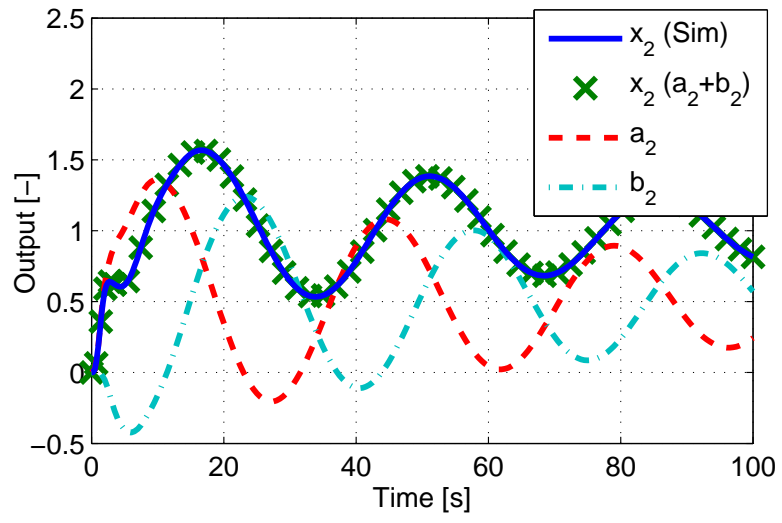

Figure 10: The comparison of the outputs of the 2nd agent simulated by the state-space approach using (88)- (89) (solid line) and that computed by the WTF approach using (90)-(93). The response to the step change of $x_{\text {ref }}(t)$ is shown.

\section{(88)-(89).}

\section{Discussion}

\subsection{Wave transfer function approach}

Let the output of the system, for instance the position, of one agent be externally changed due to an external reference command or a disturbance. Then this reference command also affects outputs of the neighbouring agents. We can imagine this command as a 'wave' propagating in the system. We illustrate the effect of the external reference by step change of $x_{\text {ref }}(t)$ in Fig 5. Since we assume a path-graph topology, the wave can travel only in two directions, which is denoted as $A$ and $B$ components. The sum of these two components, that is a wave, is then the output of the agent.

The closed form formula for the transfer function among the outputs of the agents can be found by the traditional ap- proach, for instance, by the state-space description of the multiagent system. As an example, we can find the transfer function $T_{1,2}(s)=X_{2}(s) / X_{1}(s)$ from the output of the first agent to the output of the second agent. However, to find this transfer function, the whole multi-agent system must be taken into consideration. This transfer function $T_{1,2}(s)$ then describes the overall behaviour of the multi-agent system since all the interactions among agents and, in addition the effect of boundary conditions are captured in this transfer function. This 'overall' description is well suited for determining the asymptotic stability of a system but it does not reveal the 'local' behaviour of the agents.

On the other hand, the transfer function $A_{2}(s) / A_{1}(s)$, or $B_{1}(s) / B_{2}(s)$, describes the interaction among the first and second agents from the 'local' perspective, which means that it does not take into consideration neither the interactions among other agents nor the effect of the boundary conditions. The closed loop formulas describing this 'local' interaction are given in Theorems 1 and 2. The amplitude of the wave that is reflected or transmitted through the boundary can be approximated by the DC gain of the transfer functions, which is treated by Lemma 2 and discussed in Section 5.2. The description of this local interaction is a novel in analysing and control of a multi-agent system and it it the main contribution of the paper.

An important feature of the 'local' transfer functions, i.e. the Wave or Boundary transfer functions, is that they can also be used to provide the overall transfer function $X_{2}(s) / X_{1}(s)$ by taking $\left[A_{2}(s)+B_{2}(s)\right] /\left[A_{1}(s)+B_{1}(s)\right]$. It can be shown on the examples that this transfer function has no square root, so it is rational and is equal to $T_{1,2}(s)$, which is numerically verified in Fig. 10 However, this calculation again requires to consider interactions among all the other agents, which is rather complicated with the wave approach.

Therefore, the wave approach is to be a complementary tool to the traditional state-space approach, since the wave approach gives insight into local behaviour of a multi-agent sys- 
tem, which is particularly useful for a large-scale multi-agent system.

\subsection{Wave controllers}

The advantage of the wave controller is that it allows the modification of the reflection conditions for the travelling waves on a boundary. Importantly, this modification does not require to change controllers of the other agents in the system and, under certain conditions, it can make the system string stable, as Theorem 5 shows. Another advantage is that the output of the controller is feasible as Fig. 7 shows.

The disadvantage is that the agent with the wave controller is required to know its own output, for instance, from the odometry, and the output of its immediate neighbour, which can be obtained either by the relative measurement or communication with the neighbour. Another difficulty is that the agent requires to know its own and neighbour's dynamical models. If these conditions are not satisfied, for instance, the dynamical model of neighbours is known only approximately, or the information about the neighbour's output is delayed in timep, then the wave is not fully absorbed and it partially reflects back. However, the numerical simulations show that the response of the system may still be improved since these schemes are relatively 'robust' to the inaccuracies. This is in agreement with experience from practical implementation of the wave absorbers, see for instance [28], [13] or [24].

We consider the wave controllers as an illustration what can be achieved by the application of the wave approach. We note that, although some simpler controllers may improve the transient of a system, only the wave absorber fully absorbs the travelling wave.

\section{Conclusions}

This paper introduces a local approach to a multi-agent system with path-graph interaction topology. It mathematically describes two basic types of boundaries in a multi-agent system with non-identical agents and their effect on the waves travelling in the system. The wave description allows us to design a feedback controller to compensate the effect of the boundaries, which shortens the settling time of the multi-agent system. Moreover, such a controller makes the multi-agent system string stable provided that the system is equipped with at least one wave absorber on the first or rear-end agents.

\section{Acknowledgements}

This work was supported by Grant Agency of the Czech Republic within the project GACR P103-12-1794.

The authors thank Kevin Fleming for proofreading of the manuscript.

\section{Appendix A. Proof of Lemma 3}

The proof is based on [5] (Theorem A.2), which states: $A$ linear system is stable if and only if its transfer function $T(s)$ is analytic in the right-half plane and $\|T\|_{\infty}<\infty$, where $\|T\|_{\infty}=$ $\sup _{\operatorname{Re}(s)>0}|T(s)|$.

It was proved in [17] that the WTF defined by (9) satisfies $\|G\|_{\infty} \leq 1$. Hence, it remains to derive the condition, when the WTF is analytic in the right-half plane.

First, we treat the square root function in 9 . In the complex function analysis, for instance p. 99-100 in [31], it is shown that the square root function $f(z)=\sqrt{z}$ is analytic everywhere, except for the non-positive real axis. Therefore, $f_{2}(\alpha)=\sqrt{\alpha^{2}-4}$ in (9) is analytic everywhere, except for $\alpha \in\langle-2,2\rangle$ on the real axis. Since $\alpha(s)=2+1 / M(s)$, we can say that $f_{2}(\alpha)$ is analytic everywhere, except for the interval $(-\infty,-1 / 4\rangle$. Due to the Maximum modulus principle (Theorem 4.5 [31]), we can evaluate the analyticity by the Nyquist plot of $M(s)$. This means that, if $M(j \omega)$ does not intersect interval $(-\infty,-1 / 4\rangle$ for $\omega \in(0, \infty)$, then the WTF is stable.

The first part of the WTF, $1+0.5 / M$, is a rational transfer function. A rational function is analytic in the CRHP if and only if it has no singularities in this plane. The only possible singularities of $1+0.5 / M$ are CRHP zeros of $M(s)$. Therefore, if $M(s)$ has no CRHP zero, then $1+0.5 / M$ is analytic. Since the difference of two analytic functions is again analytic, then the WTF is analytic and asymptotically stable under the above conditions.

\section{Appendix B. Proof of Theorem 5}

In this proof, we assume that there is only one soft boundary in a multi-agent system. However, the proof in the case with multiple soft and/or hard boundaries can be carried out analogously. We consider a multi-agent system with a path-graph topology with $M_{\mathrm{f}, i}=M_{\mathrm{r}, i}, M_{\mathrm{f}, j}=M_{\mathrm{r}, j}$, where $i=1,2, \ldots, m$ and $j=m+1, m+2, \ldots, k$. Therefore, there is a soft boundary between agents indexed as $m$ and $m+1$.

First, we prove the case with a wave absorber only on the first agent. If there is a wave absorber implemented on the first agent, then the combination of (7), 8, 68) and 69) gives

$$
\begin{aligned}
& \frac{X_{p}(s)}{X_{\mathrm{ref}}(s)}=G^{p}(s)+G^{2 m+1-p}(s) H^{2 k}(s), \text { if } p \leq m, \\
& \frac{X_{p}(s)}{X_{\mathrm{ref}}(s)}=G^{m}(s) H^{p-m}(s)+G^{m}(s) H^{2 k+1+m-p}(s), \text { if } p>m .
\end{aligned}
$$

In the alternative case of the wave absorbers implemented either on the rear-end agent, or on both the first and the rear-end agents, the output of the $p$ th agent is described by

$$
\begin{aligned}
& \frac{X_{p}(s)}{X_{\mathrm{ref}}(s)}=G^{p}(s), \text { if } p \leq m, \\
& \frac{X_{p}(s)}{X_{\mathrm{ref}}(s)}=G^{m}(s) H^{p-m}(s), \text { if } p>m .
\end{aligned}
$$


Since $G$ and $H$ are asymptotically stable, and $\|G\|_{\infty} \leq 1$ and $\|H\|_{\infty} \leq 1$, then $(\mathrm{B} .1)-(\overline{\mathrm{B} .4})$ are asymptotically stable too and have the $H_{\infty}$ norm limited regardless of the number of agents in the system. In view of Definition 2, we see that these two systems are $L_{2}$ string stable.

Now, we prove the case with no wave absorber on the first or rear-end agents. First, we show a way to find the transfer function from $X_{\text {ref }}$ to $X_{1}$ for the system with $m=3$ and $k=5$. In this case, $A_{1}=G X_{\text {ref }}-G^{7} H^{10} A_{1}$ and $B_{1}=G^{6} H^{10} X_{\text {ref }}-$ $G^{7} H^{10} B_{1}$, hence

$$
\frac{X_{1}(s)}{X_{\mathrm{ref}}(s)}=\frac{A_{1}(s)+B_{1}(s)}{X_{\mathrm{ref}}(s)}=\frac{G(s)+G^{6}(s) H^{10}(s)}{1+G^{7}(s) H^{10}(s)} .
$$

Similarly,

$$
\frac{X_{2}(s)}{X_{\mathrm{ref}}(s)}=\frac{G^{2}(s)+G^{5}(s) H^{10}(s)}{1+G^{7}(s) H^{10}(s)},
$$

and so on. For the $p$ th agent, we have

$$
\begin{aligned}
\frac{X_{p}(s)}{X_{\mathrm{ref}}(s)} & =\frac{G^{p}(s)+G^{2 m+1-p}(s) H^{2 k}(s)}{1+G^{2 m+1}(s) H^{2 k}(s)}, \text { if } p \leq m, \\
\frac{X_{p}(s)}{X_{\mathrm{ref}}(s)} & =\frac{G^{m}(s) H^{p-m}(s)+G^{m}(s) H^{k+2 m+3-p}(s)}{1+G^{2 m+1}(s) H^{2 k}(s)}, \text { if } p>m .
\end{aligned}
$$

Note that the transfer functions between two arbitrary agents can be expressed similarly.

Due to 9], we have $M /(1+2 M)=G /\left(1+G^{2}\right)$. The Nyquist criterion of stability states that if $M /(1+\lambda M)$ is stable for $\lambda \in(0,4)$, and if there are neither CRHP poles nor CRHP zeros in $M(s)$, then Nyquist curve of $M(s)$ does not encircle the point $[-1 / 4,0]$. Hence, $M(s)$ does not intersect the interval $(-\infty,-1 / 4\rangle$. Therefore, if 82 holds, then $M /(1+2 M)$ is asymptotically stable.

In view of the Nyquist criterion of stability, we can say that, if $M /(1+2 M)$ is stable, that is, if $G /\left(1+G^{2}\right)$ is stable, and if $\|G\|_{\infty} \leq 1$ and $\|H\|_{\infty} \leq 1$, then the transfer function

$$
\frac{G(s)}{1+G^{2}(s) G^{q_{1}}(s) H^{q_{2}}(s)},
$$

is asymptotically stable for $q_{1}, q_{2} \in \mathbb{N}$. Furthermore, since $G$ and $H$ are asymptotically stable, then the transfer function

$$
\frac{G(s) G^{q_{3}}(s) H^{q_{4}}(s)}{1+G^{2}(s) G^{q_{1}}(s) H^{q_{2}}(s)},
$$

is asymptotically stable for $q_{3}, q_{4} \in \mathbb{N}$. Comparing $(\mathrm{B} .10)$ with B.7) and (B.8), we can say that the transfer function between two arbitrary agents in a system with no wave absorber on the first or rear-end agents and with the control law given by Theorem 3 is asymptotically stable.

The proof for the control law from Theorem 4 can be carried out analogously. The only difference is in different powers of $G$ and $H$ in (B.1)-(B.4), B.7) and (B.8), which does not affect neither the asymptotic nor string stability.

\section{References}

[1] H. Bateman, A. Erdélyi, Tables of integral transforms, California Institute of technology. Bateman Manuscript project. A. Erdélyi, editor. W. Magnus, F. Oberhettinger, F. G. Tricomi, research associates, McGraw-Hill, New York, 1954.

[2] B. Briegel, D. Zelazo, M. Bürger, F. Allgöver, On the zeros of consensus networks, 50th IEEE Conference on Decision and Control and European Control Conference (2011) 1890-1895.

[3] C. E. Cantos, J. J. P. Veerman, Transients in the Synchronization of Oscillator Networks, eprint arXiv:1308.4919.

[4] M. Cao, S. Zhang, M. K. Camlibel, A Class of Uncontrollable Diffusively Coupled Multiagent Systems with Multichain Topologies (February 2013) (2012) 465-469.

[5] R. Curtain, K. Morris, Transfer functions of distributed parameter systems: A tutorial, Automatica 45 (5) (2009) 1101-1116.

[6] T. Dhaene, D. D. Zutter, Selection of lumped element models for coupled lossy transmission lines, Computer-Aided Design of Integrated ... (July) (1992) 805-815.

[7] S. K. Dwivedy, P. Eberhard, Dynamic analysis of flexible manipulators, a literature review, Mechanism and Machine Theory 41 (7) (2006) 749777.

[8] M. Egerstedt, S. Martini, M. Cao, Interacting with networks: How does structure relate to controllability in single-leader, consensus networks?, IEEE Control systems magazine (July) (2012) 66-73.

[9] J. Eyre, D. Yanakiev, I. Kanellakopoulos, A Simplified Framework for String Stability Analysis of Automated Vehicles*, Vehicle System Dynamics (1998) 375-405.

[10] A. P. French, Vibration's and Waves, M.I.T. introductory physics series, CBS Publishers \& Distributors, New Delhi, 2003.

[11] H. Hao, P. Barooah, Stability and robustness of large platoons of vehicles with double-integrator models and nearest neighbor interaction, International Journal of Robust and Nonlinear Control 23 (18) (2012) 20972122 .

[12] I. Herman, D. Martinec, Z. Hurak, M. Sebek, Nonzero bound on Fiedler eigenvalue causes exponential growth of $\mathrm{H}$-infinity norm of vehicular platoon, IEEE Transactions on Automatic Control 9286 (c) (2014) 1-1.

[13] E. Kreuzer, M. Steidl, Controlling torsional vibrations of drill strings via decomposition of traveling waves, Archive of Applied Mechanics 82 (4) (2011) 515-531.

[14] B. Lesieutre, Impedance matching controllers to extinguish electromechanical waves in power networks, IEEE International Conference on Control Applications (2002) 25-30.

[15] J. Lunze, A method for designing the communication structure of networked controllers, International Journal of Control 86 (9) (2013) 14891502 .

[16] D. Martinec, WaveBox (2015), MATLAB Central File Exchange (http://www.mathworks.com/matlabcentral/fileexchange/51958), Retrieved July 3, 2015.

[17] D. Martinec, I. Herman, Z. Hurák, M. Šebek, Wave-absorbing vehicular platoon controller, European Journal of Control 20 (2014) 237-248.

[18] D. Martinec, I. Herman, M. Sebek, Two-sided wave-absorbing control of a heterogenous vehicular platoon, in: Proceedings of the 19th IFAC World Congress, 2014, No. 1986, 2014, pp. 8091-8096.

[19] C. Mei, Wave control of vibrations in multi-story planar frame structures based on classical vibration theories, Journal of Sound and Vibration 330 (23) (2011) 5530-5544.

[20] M. Mesbahi, M. Egerstedt, Graph theoretic methods in multiagent networks, Princeton Series in Applied Mathematics, Princeton University Press, New Jersey, 2010.

[21] R. H. Middleton, J. H. Braslavsky, String Instability in Classes of Linear Time Invariant Formation Control With Limited Communication Range, IEEE Transactions on Automatic Control 55 (7) (2010) 1519-1530.

[22] W. J. O'Connor, Wave-Based Analysis and Control of Lump-Modeled Flexible Robots, IEEE Transactions on Robotics 23 (2) (2007) 342-352.

[23] W. J. O'Connor, Wave-like modelling of cascaded, lumped, flexible systems with an arbitrarily moving boundary, Journal of Sound and Vibration 330 (13) (2011) 3070-3083.

[24] W. J. O’Connor, F. Ramos de la Flor, D. J. McKeown, V. Feliu, Wavebased control of non-linear flexible mechanical systems, Nonlinear Dynamics 57 (1-2) (2008) 113-123. 
[25] R. Olfati-Saber, J. Fax, R. Murray, Consensus and cooperation in networked multi-agent systems, Proceedings of the IEEE (January) (2007) 215-233.

[26] J. Ploeg, N. van de Wouw, H. Nijmeijer, Lp String Stability of Cascaded Systems: Application to Vehicle Platooning, IEEE Transactions on Control Systems Technology 22 (2) (2014) 786-793.

[27] W. Ren, R. W. Beard, E. Atkins, Information consensus in multivehicle cooperative control, IEEE Control systems magazine (April) (2007) 7182.

[28] M. Saigo, N. Tanaka, D. H. Nam, Torsional vibration suppression by wave-absorption control with imaginary system, Journal of Sound and Vibration 270 (4-5) (2004) 657-672.

[29] W. Singhose, S. Derezinski, N. Singer, Extra-insensitive input shapers for controlling flexible spacecraft, Journal of Guidance, Control, and Dynamics 19 (2) (1996) 385-391.

[30] L. Sirota, Y. Halevi, Fractional order control of the two-dimensional wave equation, Automatica 59 (2015) 152-163.

[31] E. M. Stein, R. Shakarchi, Complex Analysis, Princeton lectures in analysis, Princeton University Press, New Jersey, 2010.

[32] D. Swaroop, J. Hedrick, String stability of interconnected systems, IEEE Transactions on Automatic Control 41 (3) (1996) 349-357.

[33] D. Swaroop, J. K. Hedrick, Constant Spacing Strategies for Platooning in Automated Highway Systems, Journal of Dynamic Systems, Measurement, and Control 121 (3) (1999) 462.

[34] F. Tangerman, J. Veerman, B. Stosic, Asymmetric decentralized flocks, IEEE Transactions on Automatic Control 57 (11) (2012) 2844-2853.

[35] K. Yamamoto, M. Smith, Mass chains with passive interconnection: Complex iterative maps and scalability, in: 52nd IEEE Conference on Decision and Control, 2013, pp. 37-42. 